Euskal ikerketen aldizkaria | Revue d'études basques |

Revista de estudios vascos | Basque studies review

$4 \mid 1999$

Numéro IV

\title{
Documents inédits pour l'histoire d'Anglet
}

\section{Manex Goyhenetche}

\section{OpenEdition \\ Journals}

Édition électronique

URL : http://journals.openedition.org/lapurdum/1572

DOI : 10.4000/lapurdum.1572

ISSN : 1965-0655

Éditeur

IKER

Édition imprimée

Date de publication : 1 octobre 1999

Pagination : 287-293

ISBN : 2-84127-156-0

ISSN : $1273-3830$

\section{Référence électronique}

Manex Goyhenetche, «Documents inédits pour l'histoire d'Anglet », Lapurdum [En ligne], 4| 1999, mis en ligne le 01 avril 2010, consulté le 01 février 2020. URL : http://journals.openedition.org/lapurdum/ 1572 ; DOI : 10.4000/lapurdum.1572 
Manex GOYHENETCHE

\section{DOCUMENTS INÉDITS POUR L'HISTOIRE D'ANGLET}

\section{UNE TRANSACTION A ANGLET EN 1788 : le domaine de Latchague}

(Archives départementales des Pyrénées-Atlantiques, IIIE 4736)

\section{Présentation et intérêt du texte}

Cet acte notarié constitue un matériau d'étude, dans une interdisciplinarité de bon aloi, aussi bien pour le linguiste, l'anthropologue que l'historien. Voici, suggérées, à grands traits, quelques pistes de recherche

* Intérêt pour le linguiste. Comme c'est très souvent le cas à Anglet, pour les documents d'époque moderne, toponymes et anthroponymes d'origine basque et gasconne s'entremêlent : "Vignaux", "Sablot" ou "Dou Bigné" d'un côté, Latxague, Dhiriart ou Darancette de l'autre. Actuellement, le domaine de Latchague appartient aux Servantes de Marie.

En complément, on peut ajouter que le domaine attenant, appelé "Lohiate", avait été aussi acheté par les Servantes de Marie, au XIX ${ }^{e}$ siècle, au sieur de Châteauneuf, autre propriétaire bayonnais. Le toponyme "Lohiate", d'origine basque, a disparu dans la documentation actuelle. Pourtant, c'est sous ce nom qu'il apparaît, par exemple, dans la carte de Cassini (XVIII ${ }^{e}$ siècle). Le 5 Ventôse An II (23 février 1794), le conseil de la commune d'Anglet, pour répondre à l'effort de guerre demandé par la Convention, recensait parmi les "portails en fer de pur luxe" celui de Lohiate appartenant au "citoyen Pierre Chateauneuf propriétaire du bien appelé Lohiatte de cette commune". Aux linguistes de restituer l'étymologie de ce terme. Mais, il est instructif de savoir que le ruisseau "Maharin" ("Méharin"), autre toponyme basque, prend sa source au même endroit.

* Intérêt pour l'anthropologue. Au vu de ce document, il semble qu'existaient des rites de prise de possession des biens. Le nouvel acquéreur se rendait dans la propriété dont il était désormais le maître et, par des actes symboliques, signifiait la "possession réelle", en prenant une poignée de chaque sorte de biens, en l'occurrence "une poignée de terre, d'herbes, légumes et branches et feuillages de bois". Quelle était 1'étendue géographique d'une telle pratique ? On la trouve, par exemple, dans les pièces d'une procédure de la famille Duhalde à Ustaritz concernant une succession (voir en Arch. dép. des Pyr.-Atl., 1 J 284/1-29).

* Intérêt pour l'historien. Étienne Moulia et le chevalier de Salha sont des personnages représentatifs de l'ascension sociale de familles bayonnaises, bourgeoises (Moulia) ou d'ancienne noblesse (Salha) qui exerçaient une emprise ou 
une pression foncière sur la campagne environnante, en l'occurrence Anglet. La consultation des archives de cette commune montre, qu'outre Moulia et Salha, d'autres Bayonnais possédaient des biens à Anglet, notamment Sorhainde, Chavenau, Dordos, Ducassou, Saboulin, David, Cabarrus, Lesseps, Poydenot, Boby, appelés par les gens d'Anglet “sieurs bourgeois". Une exploitation systématique des archives notariales devrait permettre de mesurer et de quantifier l'importance de l'installation dans la campagne angloye des bourgeois et des robins d'origine bayonnaise.

Aujourd'hui huitième du mois de juin mil sept cens quatre vingts huit, après midi, en la paroisse d'Anglet, pais de Labourt, par devant nous Notaire Royal de ladite parroisse soussigné, présens les témoins bas nommés, est comparu Bernard Lafitte vigneron habitant de la présente parroisse, quartier de Brindos, lequel de sa libre et franche volonté a vendu, quitté, cédé, délaissé et transposé ainsi que par ces présens il vend, quitte, cède, délaisse et transporte purement et simplement sans aucune espèce de réserve, ni sans autre ni plus ample garantie que celle qu'il a de ses vendeurs, au sieur Etienne Moulia premier huissier de la Bourse de Bayonne, demeurant en ladite Ville, actuellement ici présent, stipulant et accepiant, acquéreur pour lui, ses successeurs et ayants droit et cause à l'avenir : à savoir est tout l'héritage appellé Latchague, ensemble les deux métairies en dépendantes appellées Vignaux et Sablot, situées en ladite présente paroisse d'Anglet, consistant en maison de Maitre, et trois autres différentes maisons, cours, parcs à bétail, pressoirs, jardins, terres labourables, hautins, bois, échalassierr's, terres, landes et autrement dits communaux de partage, prairie, et généralement toutes les appartenances et dépendances desdits trois héritages en quoique le tout puisse consister sans aucune sorte d'exception ni de réserve. comme aussi et par exprès la pièce de terre communément appellée de Salleneuve qui fait actuellement une dépendance de Latchague, depuis qu'elle a étí défrichée et mise en valeur en l'année mil sept cens quatre vingts six, et autre pièce de terre d'une journade, tenue en engagement des dépendances de la maison appellée dou Bigné, ensemble toutes entrées, issues, servitudes, droits, noms, raisons et actions rescindes et rescisoires, droits d'église et de sépulture et absolument tout ce que comporte, contient et dépend desdits trois héritages, ainsi et d'autant que ledit Lafitte vendeur en a acquis de dame Anne Luce d'Urdos, épouse de Messire Valentin, Chevalier de Salha, Lieutenant des vaisseaux du Roi, autorisée par lui et de son avis, consentement et garantie expresse, suivant le contrat sur ce passé le quatre du présent mois devant Maître D'Hiriart notaire royal du présent lieu. Observant cependant, de même qu'il est porté et fait mention au susdit contrat, que le bois de Latchague est absolument dépeuplé d'arbres de haute futaye, depuis que l'année dernière mondit sieur de Salha les en a fait ôter pour être employés à la construction de son hôtel d'Artois situé à Bayonne, me du Gouvernement, francs, quittes et libres, exempts et affranchis lesdits trois héritages et ci-dessus vendus de toutes dettes, charges et hipotèques, généralement quelconques de tout le passé jusqu'à ce jour, dont si aucuns y a ou peut avoir ledit Lafitte subrogé et met à son lieu et place ledit sieur Moulia acquéreur, pour par lui exercer et faire valoir contre lesdits Sieur et Dame de Salha ou leurs représentants à l'avenir la promesse, obligation et garantie stipulée pour raison de ce dans le susdit contrat du quatre du présent mois, ce que le Sieur Moulia a accepté et accepte. 
Laquelle vente est ainsi faite et convenue pour et moyennement le prix et somme de douze mille livres, sur et en déduction de laquelle ledit Sieur Moulia a payé, compté et réellement déléivré audit Lafitte, avant ces présens, ainsi que celui-ci en est convenu et demeuré d'accord devant nous dit Notaire et témoins la somme de trois mille livres en écus de six livres pièce, et de laquelle somme de trois mille livres ledit Lafitte a déclaré être pleinement content et satisfait et en acquitter et décharger purement et simplement ledit Sieur Moulia, le subrogeant même par exprès en son lieu, droit, place, privilège spécial et hipotèque pour raịson de pareille somme comptée par ledit Lafitte à mesdits Sieur et Dame de Salha, lors de l'aquisition par lui faite des héritages ci-dessus rendus et laquelle dite somme a alors passé de la main à la main au pouvoir du Sieur Lissalde Maître maçon et Lavie Maître charpentier ouvriers employés à la batisse dudit hôtel d'Artois, qui à leur tour ont subrogé et mis ledit Lafitte à leur lieu et place.

Et à l'égard des neuf mille livres restantes, pour compléter ledit prix de vente, il a été convenu et accordé entre ledit Lafitte vendeur et ledit Sieur Moulia acquéreur que celui-ci prend et assume sur lui la condition faite entre ledit Lafitte et mesd. Sieur et Dame de Salha par ledit conrat du quatre de ce mois. En conséquence, ledit Sieur Moulia promet et s'oblige de payer et acquitter à la décharge de ladite Dame d'Urdos de Salha, $1^{\circ}$ aux Dames religieuses de Sainte Ursule de la ville Dax la somme de trois mille livres en capital par elle due à leur communuté comme cessionnaire d'une demoiselle de Luppé suivant un ancien contrat de constitution de rente hipotéqué sur les biens immeubles de la Dame d'Urdos de Salha à elle obtenus du chef du feu Sieur Joseph Sorhainde son oncle maternel, $2^{\circ}$ celle de six mille livres de capital également due à la Dame Labarthe veuve Lamagnère suivant un contrat d'obligation dudit jour quatre de ce mois, retenu de Me D'Hiriart notaire Royal à Bayonne, dont les deniers ont servi à payer jusqu'à cette concurrence le maçon employé à la construction de l'Hôtel d'Artois, appartenant à ladite Dame d'Urdos de Salha et situé à Bayonne; le remboursement de laquelle somme est convenu et fixé par parcelles de deux mille livres chacune, payables dans trois, six et huit années; et en attendant sa libération, ledit Sieur Moulia promet et s'oblige de payer soit aux dites Dames religieuses Sainte Ursule Dax, et soit à la Dame Labarthe veuve Lamagnère l'intérêt qui les concerne suivant l'énoncé en leurs contrats.

Et comme les maisons, dépendances des héritages ci-dessus vendus sont totalement détruits par vétusté, que certaines parties ont croulé et que d'autres menacent une ruine prochaine, et que ledit Lafitte a fait détailler amplement cet article dans son contrat, afin de prendre ses précuations lors des réédifications ou réparations urgentes à cause de l'incertitude où l'on étoit sur l'Etat libre de ces biens et assurer une solide répétition des dépenses à faire ce concernant, ledit Lafitte déclare par exprès subroger en son lieu et plce ledit Sieur Moulia acquéreur dans l'objet énoncé audit contrat du quatre de mois et relatif à l'état de délabrement desdites maisons et aux précautions à prendre pour les faire réparer; consentant qu'il fasse tel usage qu'il jugera à propos de l'énoncé en ladite clause et qu'il en tire tout le fruit et avantage qui pourra en rsulter, le lsuborogeant généralement quantà ce en son dit, lieu, place, prilège et hipotèque.

Au moyen de tout ce dessus ledit Sieur Lafitte s'est démis et dépouillé de la propriété, possession et jouissance, tant dudit héritage de Latchague que des deux métairies en dépendantes et de tous autres objets ci-dessus vendus, et en a 
vétu et saisi ledit Sieur Moulia, consentant qu'il en use, jouisse, fasse et disposer à son plaisir et volonté et comme de chose à lui propre, bien et légitimement acquise, et qu'il en fasse tous les fruits siens depuis ce jour, comme aussi qu'il en prenne la possession réelle, actuelle et corporelle quand bon lui semblera, ledit Lafitte présent ou absent et sans qu'il soit besoin de l'y appeller, lui en donnant la verballe par la remise d'une expédition en forme du contrat de vente dudit jour quatre de ce mois dont lecture a été en l'instant faite en présence desdits témoins.

Et pour l'entretennement et exécution de tout ce dessus lesdites parties ont obligé, affecté et hipotéqué tous leurs biens meubles et immeubles présens et à venir chacune à son égard, et spécialement ledit sieur Moulia lesdits biens pr lui acquis sans que l'hypotèque spéciale puisse déroger à la générale, et iceux soumis aux rigueurs de la justice.

Fait et passé en présence de Sieurs Saubat Ducassou et Pierre Dubroca laboureurs habitans de cette parroisse d'Anglet, témoins à ce requis et signés à l'original avec ledit Sieur Moulia, ce que n'a fait ledit Sieur Lafitte pour ne savoir écrire ainsi qu'il la déclaré de ce faire interpellé par nous dit Notaire.

Darancette Notaire Royal

Et le treisième du mois de juin mil sept cens quatre vingts huit après midi en ladite parroisse d'Anglet pais et Bailliage de Labourt, par devant moi Notaire Royal soussigné présens les témoins bas nommés, a comparu Sieur Étienne Moulia premier huissier de la Cour de la Bourse de Bayonne y habitant et tomicilié, lequel a dit qu'il auroit acquis de Bernard Lafitte vigneron de cette parroisse d'Anglet y habitant au quartier de Brindos, par le présent contrat de vente du huit de ce mois, retenu par moi dit Notaire tout l'héritage appellé Luichague ensemble les deux métairies en dépendantes appellées Vignau et Sablot, situées en cette parroisse, consistant en maison de maitre et trois autres maisons, cours, parcs à bétail, pressoirs, jardin, terres labourables, hautin, bois échalassière, landes et prairie, situés aussi au présent lieu et désirant en prendre possession, il auroit requis moi dit notaire et vouloir l'y installer. Pour y satisfaire, je dit Notaire en compagnie desdits Sieurs Moulia, Lafitte à ce présent et consentant et des témoins me suis transporté dans lesdits héritages maisons de Latchague, Vignau, Sablot et dans les autres trois maisons, c'est-à-dire en tout dans les susdites quatre maisons, cours, parcs à bétail et pressoir où étant aurions trouvé les nommés Pierre Farron, Étienne Duffourcq et Pierre Laporterie, fermier, métayer et locatare d'icelle et auroit pris la possession réelle, actuelle et corporelle et en signe d'une vraye et réelle possession, il auroit ouvert et fermé les portes d'icelles maisons, allumé et éteint les feux et touché les crémaillères, ensuite de quoi serions en suite transportés sur toutes les susdites terres, jardin, hautins, bois échalassières, prairies et landes et sur chacun d'iceux il auroit pris et jetté une poignée de terre, d'herbes, légumes et branches et feuillages de bois, ainsi que celles que nous lui avons mis en main, et tant dans les susdites maisons que les susdits biens énoncés audit contrat, auroit fait et observé tous les autres actes possessoires et tant dans les susdites maisons que les susdits biens énnoncés audit contrat, auroit fait et observé tous les autres actes possessoires et formalités à ce requis et nécessaires aussi en présence desdits fermier, métayer et locataire, auxquels aurions enjoint de reconnoitre désormais comme depuis ledit 
contrat, le Sieur Moulia acquéreur pour propriétaire incommutable desdites maison de Latchague et des trois autres maisons et des biens, à quoi ils auroient promis de se conformer. Au surplus ledit Sieur Moulia auroit resté et se seroit promené tant dans lesdits héritage et maisons que biens en dépendants tout autant de temps qu'il a jugé à propos au vu et sçu de tous ceux qui l'ont voulu voir et savoir sans que personne ait rien dit ni opposé. De quoi ledit Sieur Moulia a requis à moi dit notaire de retenir acte. Ce que je lui ai octroyé pour servir et valoir ce que de raison. Fait en présence de Sieur Jean Bernainn marchand relieur dudit Bayonne y Habitant, Pierre Dubroca et Étienne Gimon, laboureurs habitans de cette parroisse d'Anglet, témoins à ce requis et signés à l'original avec ledit Sieur Moulia, ce que n'ont fait lesdits fermier, métayer et locataire ni ledit Lafitte, pour ne savoir écrire ainsi qu'ils ont déclaré de ce faire, interpellés par moi.

Darancette notaire Royal

\section{CONTRAT DE FERME}

\section{DES ADJUDICATIONS DE L'IMPÔT SUR LE VIN}

(Archives départementales des Pyrénées-Atlantiques III E 4732)

\section{Présentation et intérêt du texte}

La ferme du vin ou mayade était une sorte de taxe à la consommation. Elle constituait, avec celle de la viande, l'essentiel de la fiscalité indirecte dans le budget communal. Elle prenait effet à la Saint-Martin et les adjudications avaient lieu en novembre, mais pouvaient se prolonger jusqu'à décembre si l'adjudication ne rapportait pas assez. L'assemblée affermait le revenu à des particuliers appelés fermiers, moyennant une somme annuelle déterminée par offre publique ou adjudication. La somme versée par le fermier était une avance sur la taxe ; à lui ensuite de la recouvrer conformément à des critères que fixait l'assemblée paroissiale. Le fermier adjudicataire signait obligatoirement un contrat avec les jurats, devant notaire. Nous disposons de peu de documents sur les actes notariés de ce genre. Dans le cas actuel, l'adjudicataire est "Joannes Dolhagaray, forgeron". Le document permet de connaître dans le détail les conditions et les contraintes mises en place pour le commerce et la vente du vin.

"14 décembre 1783 : Mayade ferme des impositions en faveur de Joannes Dolhagaray maître forgeron, Sieur jeune de la maison de Partaire

La communauté donne permission d'en vendre les vins qui se débiteront dans l'étendue de cette parroisse pendant une année qui a cours depuis le huit de ce mois et à finir à pareil jour huitième de décembre de l'année prochaine

Ledit Dolhagaray promet et s'oblige de payer audit Sieur Hirigoyen ou ordre pareille somme [...] au moyen de quoy ledit sieur Hirigoyen a mis et met led. Sieur d'Olhagaray fermier en possession de percevoir aud. nom de la communauté lesd. impositions consistants sçavoir de chaque barrique de vin du cru du lieu dudit Anglet cinq livres, de chaque barrique de vin Etranger huit livres et 
chaque bouteille de vin d'Espagne quarante sols. Led. fermier a promis et s'est obligé de pourvoir les habitants de bon vin loyal et marchand dans quatre chaïs qu'il établira sçavoir l'un au quartier de Haut, l'autre au quartier de Bas, l'autre à celuy de Brindos et l'autre au quartier de Sutar suivant l'usage pratiqué dont lad. débite sera faite au prix qu'il pourra jusques à ce que la communauté ait fait une taxe fixe ce que led. Sieur abbé a réservé pour la Communauté laquelle fourniture sera faite dans chacun desdits chaïs à peine de cinquante livres pour chaque contravention laquelle sera exigée par la voye de la pignore de l'autorité des sieurs abbé et jurats en charge aud. lieu suivant l'usage pratiqué et appliquée la moitié à la réparation de l'église et l'autre moitié aux affaires de la dite communauté bien entendu qu'il sera permis auxd. habitants de vendre et de débiter du vin durant cette ferme en payant néanmoins l'impos aud. fermier sur le pied fixé et avant de mettre tel vin en perte à peine de dix livres contre ceux qui $y$ conviendront pour chaque fois la peine ira au proffit dud. fermier et sera exigée par lesd. sieurs abbé et jurats par pignore étant convenu que le vin qui pourra être vendu par des particuliers dans leurs maisons même de leur crû sera sujet à même imposition exigé comme dessus, de plus il est arrêté entre les parties que les cabaretiers détaillans de tel vin dans l'étendue de la paroisse durant cette ferme seront teneus avant d'enchayer du vin destiné à détailler d'avertir led. fermier à peine de confiscation et de cinquante livres d'amende apliquée sçavoir la moitié pour la communauté et l'autre aud. fermier, exigée par la voie de la pignore, par lesd. Sieurs abbé et jurats ; il est de plus statué qu'il ne sera pas permis aux habitants de porter du vin ni faire porter pour être revendu en bouteille à peine de dix livres et de confiscation exigée suivant l'usage ordinaire par les Sieurs abbé, jurats et appliquée au proffit dud. fermier; sera néanmoins permlis à tous particuliers habitant de cette parroisse durant cette ferme d'envoyer chercher du vin Etranger pour leur provision seulement sans aucune permission dudit fermier soit en bouteilles, barriques ou demy barriques sans rien payer aud. fermier bien entendu cependant qu'il demeure deffendu à peine de confiscation aux étrangers d'introduire dans cette paroisse desdits barrils et bouteilles du vin étranger sans permission dud. fermier. Il sera permis à tous habitans de vendre $d u$ vin d'Espagne aux prix qu'ils pourront en payant l'impos de quarante sols par. bouteille, à la charge par eux de le dénoncer avant de l'enchayer à peine de confiscation de dix livres, icelle peine appliquée au proffit dud. fermier et exigée par lesd. Sieurs abbé et jurats, par pignore et pour plus de sureté des obligations cy dessus stipulées de la part dud. fermier soit les pour les sus des payements aux sus dits délaï".

\section{CANDIDATURE POUR UNE FERME-ÉCOLE A LAUSUCQ (SUTAR)}

\section{Présentation et intérêt du texte}

L'année 1848 fut bien mouvementée en France. Après les journées révolutionnaires des 23-24 février, un gouvernement provisoire fut institué. En mai, la république fut proclamée, puis, au début de l'été, la révolte révolutionnaire noyée dans le sang et la répression. Le 12 novembre, la présidence de la République fut instituée. En ce milieu du XIX ${ }^{\mathrm{c}}$ siècle, pour sortir la campagne des pesanteurs tant 
sociologiques que techniques, des fermes-modèles furent impulsées. Dans le département des Basses-Pyrénées, trois communes firent acte de candidature, en 1848, pour accueillir la ferme-école : Escos, Gan et Anglet. Au vu des articles contenus dans Le Mémorial des Pyrénées, les rapports de la commission d'examen ne retinrent pas la candidature de la ferme Laussucq, située au quartier de Sutar à Anglet. Dans le cadastre de 1830, le propriétaire en était Michel Hiriart, originaire de Bayonne.

\section{Articles contenus dans Le Mémorial des Pyrénées}

30 novembre 1848

Compte rendu du Conseil Général des Basses-Pyrénées. Séance du 29 novembre :

[...] Monsieur Laborde, autre rapporteur, présente au nom de' la quatrième commission (Administration Générale) un rapport sur les fermes-écoles. Trois propositions ont été faites pour la ferme-école. La première émane de Monsieur d'Andurain, propriétaire, à Lagarde d'Escos; la seconde, dans les mêmes conditions, de Monsieur Chauviteau, à Gan; la troisième, de Monsieur Etcheverry, élève de Grignon et fermier de Lausucq, près d'Anglet. Il n'a pas été possible à l'administration de faire des vérifications sur ces domaines.

Le Conseil n'a pas de données certaines. La Commission a donc pensé qu'elle ne pouvait se prononcer en connaissance de cause, qu'il convenait d'inviter Monsieur le Préfet à appeler les propositions par la publicité et à faire vérifier les domaines offerts".

26 janvier 1849

"Sur neuf domaines proposés [...] la commission a présenté deux ex aequo, celui de Lagerde à Escos, appartenant à Monsieur d'Andurain, et celui de Monsieur Chauviteau à Gan [...]".

(Document transmis aimablement par Jean-Pierre Dugène)

Manex GOYHENETCHE

(Docteur en Histoire) 